\title{
Field-Induced Magnetization Steps in Intermetallic Compounds and Manganese Oxides: The Martensitic Scenario
}

\author{
V. Hardy ${ }^{1,2}$, S. Majumdar ${ }^{1}$, S. Crowe ${ }^{1}$, M. R. Lees ${ }^{1}$, D. Mc K. Paul ${ }^{1}$, L. Hervé ${ }^{2}$, \\ A. Maignan ${ }^{2}$, S. Hébert ${ }^{2}$, C. Martin ${ }^{2}$, C. Yaicle ${ }^{2}$, M. Hervieu ${ }^{2}$ and B. Raveau ${ }^{2}$ \\ (1) Department of Physics, University of Warwick, \\ Coventry, CV4 7AL, United Kingdom. \\ (2) Laboratoire CRISMAT, UMR 6508, \\ Boulevard du Maréchal Juin, \\ 14050 Caen, France.
}

\begin{abstract}
Field-induced magnetization jumps with similar characteristics are observed at low temperature for the intermetallic germanide $\mathrm{Gd}_{5} \mathrm{Ge}_{4}$ and the mixed-valent manganite $\mathrm{Pr}_{0.6} \mathrm{Ca}_{0.4} \mathrm{Mn}_{0.96} \mathrm{Ga}_{0.04} \mathrm{O}_{3}$. We report that the field location -and even the existence- of these jumps depends critically on the magnetic field sweep rate used to record the data. It is proposed that, for both compounds, the martensitic character of their antiferromagnetic-to-ferromagnetic transitions is at the origin of the magnetization steps.
\end{abstract}

PACS numbers: 75.60.Ej, 81.30.Kf, 75.80.+q

The $\operatorname{Gd}_{5}\left(\mathrm{Si}_{x} \mathrm{Ge}_{1-x}\right)_{4}$ pseudobinary system has attracted a growing interest in recent years 1.2.3.4.5.6.7.8.9 owing to the wealth of interesting physical properties it displays including a giant magnetocaloric effect $\underline{\underline{1}}$ and a colossal magnetostriction ${ }^{2}$ These striking phenomena are related to a strong interplay between the magnetic and the structural features in this system. These compounds have a layered structure made up of subnanometric slabs connected via covalent-like bonds $\underline{\underline{?}}$ The degree of interslab connectivity not only depends on $x$ but also on the magnetic state. For instance, with $x=0$, the slabs are completely interconnected in the ferromagnetic (FM) state, whereas all the bonds are broken in both the antiferromagnetic (AF) and paramagnetic $(\mathrm{P})$ states 6

Recently, Levin et al s $^{8}$ have reported an intriguing phenomenon for $\mathrm{Gd}_{5} \mathrm{Ge}_{4}$. After zero-field-cooling (ZFC), the field-increasing branch of $M(H)$ curves recorded at low$T$ exhibit an extremely sharp, irreversible magnetization step. It has been proposed that this behavior is related to the strongly anisotropic exchange interactions present in this material. One should note, however, that the observation of such sharp steps in polycrystalline samples is quite unusual for conventional metamagnetic transitions.

Interestingly, similar magnetization steps were recently observed for mixed-valent manganese oxides with the general formula $\mathrm{Pr}_{1-x} \mathrm{Ca}_{x} \mathrm{Mn}_{1-y} \mathrm{M}_{y} \mathrm{O}_{3}$ (with $x \sim 0.5, y$ $\sim 0.05$, and where $\mathrm{M}$ is a cation used to destabilize the Mn-sublattice) ${ }^{10,11.12}$ The Mn-site substitutions weaken the robust CE-type AF ordering of the parent compound $\mathrm{Pr}_{1-x} \mathrm{Ca}_{x} \mathrm{MnO}_{3}(x \sim 0.5)$, and favor the development of a phase separation between FM and AF domains. Owing to the collective orbital ordering (OO) accompanying the AF spin ordering, the unit cell of the AF phase is strongly distorted with respect to that of the FM phase. Therefore, as a magnetic field is applied, competition develops between the magnetic energy promoting the development of the FM phase and the elastic energy associated with

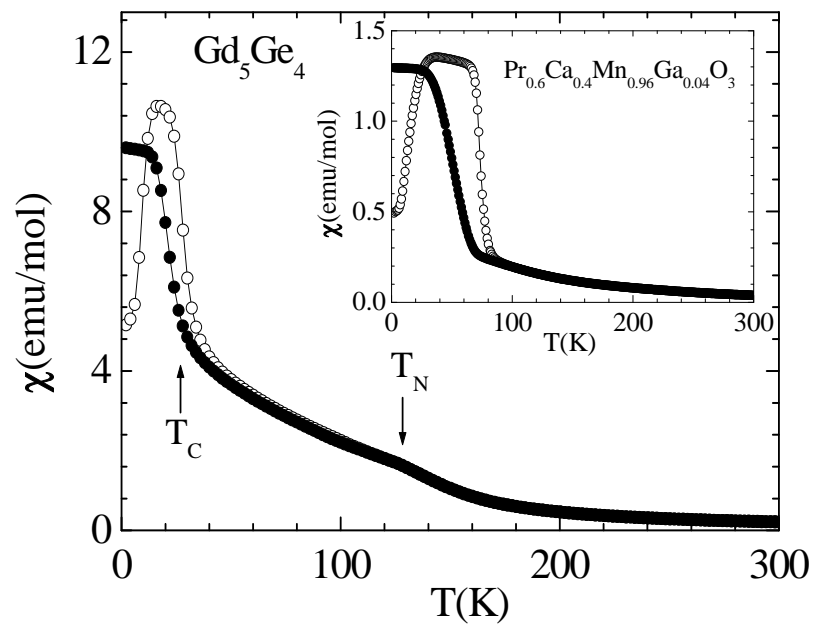

FIG. 1: $d c$ susceptibility curves recorded for $\mathrm{Gd}_{5} \mathrm{Ge}_{4}$ (main panel) and $\mathrm{Pr}_{0.6} \mathrm{Ca}_{0.4} \mathrm{Mn}_{0.96} \mathrm{Ga}_{0.04} \mathrm{O}_{3}$ (inset) in a field of 1.2 T. The open and closed symbols correspond to the zero-field cooled and field cooled cooling modes respectively. Arrows and labels on the main panel denote the Néel temperature $\left(T_{N} \sim 127 \mathrm{~K}\right)$ and Curie temperature $\left(T_{C} \sim 25 \mathrm{~K}\right)$ of $\mathrm{Gd}_{5} \mathrm{Ge}_{4}$

the strains created at the AF/FM interfaces, which tends to block the transformation 13 The martensitic nature of this transformation has led us to propose that the magnetization step corresponds to a burst-like growth of the FM component when the driving force overcomes the energy barriers associated with the strains. Remarkably, it turns out that $\mathrm{Gd}_{5} \mathrm{Ge}_{4}$ is also a system in which FM and $\mathrm{AF}$ domains can co-exist, and the transformation between these two phases has a pronounced martensitic character $\stackrel{6.8}{6}$ This is due to the collective shear movement of the slabs at the AF/FM transition which produces a considerable distortion of the unit cell. For the closely related $\mathrm{Gd}_{5}\left(\mathrm{Si}_{0.1} \mathrm{Ge}_{0.9}\right)_{4}$ compound, Morellon et al $\mathrm{l}^{\underline{3}}$ re- 


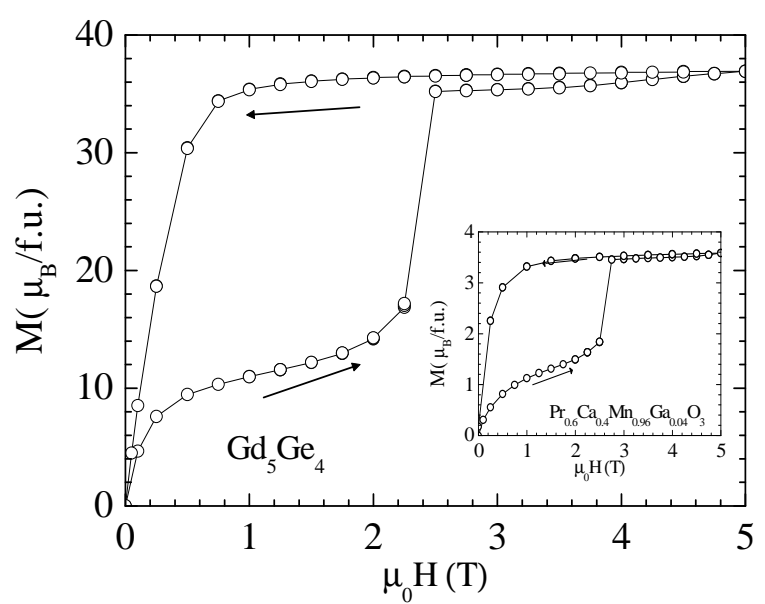

FIG. 2: Magnetic hysteresis loops recorded with a SQUID magnetometer after zero-field cooling: at $2 \mathrm{~K}$ for $\mathrm{Gd}_{5} \mathrm{Ge}_{4}$ (main panel), and at $3.25 \mathrm{~K}$ for $\mathrm{Pr}_{0.6} \mathrm{Ca}_{0.4} \mathrm{Mn}_{0.96} \mathrm{Ga}_{0.04} \mathrm{O}_{3}$ (inset)

ported that the cell parameter $a$ decreases by $1.6 \%$, while $b$ and $c$ increase by 0.7 and $0.3 \%$, respectively, at the AF/FM transition (Pnma space group for both phases).

This set of features has prompted us to undertake a precise comparative study between $\mathrm{Gd}_{5} \mathrm{Ge}_{4}$ and a $\mathrm{Mn}$ site substituted manganite, with a particular focus on the influence of the magnetic field sweep rate on the field-induced transformations. Our goal was to further investigate the similarity of the magnetization steps in these two systems and the relevance of the martensitic scenario to both of them.

The $\mathrm{Gd}_{5} \mathrm{Ge}_{4}$ sample was prepared by arc melting a stoichiometric mixture of $99.9 \mathrm{wt} \%$ pure Gd and 99.99 wt\% pure Ge. The synthesis was carried out under a high-purity argon atmosphere, turning the sample several times to ensure a good homogeneity. The Gd/Ge ratio was checked by EDS (energy dispersive spectroscopy) to be equal to the nominal composition to within the accuracy of this technique. No impurities were detected by x-ray powder diffraction, which showed that the system has an orthorhombic structure at room temperature (Pnma space group) with lattice parameters $[a=7.68(1) \AA, b=14.80(1) \AA$ and $c=7.77(1) \AA]$ in line with the literature $\underline{6}$ The manganite chosen to compare with $\mathrm{Gd}_{5} \mathrm{Ge}_{4}$ is $\mathrm{Pr}_{0.6} \mathrm{Ca}_{0.4} \mathrm{Mn}_{0.96} \mathrm{Ga}_{0.04} \mathrm{O}_{3}$, hereafter denoted as $[\mathrm{PrCa} 40] \mathrm{Ga} 4 \%$. This compound exhibits a phase-separation similar to that of $\mathrm{Gd}_{5} \mathrm{Ge}_{4}$, and it is less sensitive to training effects than most of the manganites 11 A [PrCa40] Ga4\% ceramic sample was synthesized by solid-state reaction according to a process described previously 10 It also has a room-temperature Pnma orthorhombic structure with $a=5.4293(3) \AA$, $b=7.6443(4) \AA$ and $c=5.4097(3) \AA$. Magnetic measurements were carried out using a Superconducting Quantum Interference Device (SQUID) magnetometer and a
Vibrating Sample Magnetometer (VSM). All the $M(H)$ curves have been recorded after being ZFC from the paramagnetic state at $300 \mathrm{~K}$.

The main panel of Fig. 1 shows the ZFC (Zero Field Cooled) and the FCC (Field Cooled Cooling) $d c$ magnetic susceptibility $(\chi)$ curves as a function of temperature for $\mathrm{Gd}_{5} \mathrm{Ge}_{4}$. These curves were recorded in a field of 1.2 $\mathrm{T}$ to be comparable with those of Ref. 8 . The inset shows the same data sets for $[\mathrm{PrCa} 40] \mathrm{Ga} 4 \%$. The $\chi(T)$ curves of $\mathrm{Gd}_{5} \mathrm{Ge}_{4}$ exhibit the same general features as those reported by Levin et al $\underline{\underline{\underline{8}}}$ : (i) a kink at $T_{N}=127$ $\mathrm{K}$, and (ii) an increase of $\chi$ at low- $T$, which is associated with the onset of a FM ordering. There is a pronounced hysteresis at low- $T$ that points to the first-order character of this ferromagnetic transition, the inflection point on the ZFC and FCC curves being at $\sim 28$ and $\sim 21 \mathrm{~K}$, respectively. As in Ref. 8, one can also observe that the maximum value of $\chi(T)$ is larger for the ZFC data than for the FCC data, and that there is a steep rise of the ZFC $\chi(T)$ on the low- $T$ side of the peak. It should be noted that this last feature is quite unusual for standard ferromagnets in a field as large as 1.2 T. It suggests that the onset of the FM phase is hindered when zero-field cooling this compound down to very low temperature. In addition, note that the difference between the values of the magnetization for the ZFC and FCC curves around $20 \mathrm{~K}$ may be related to the large magnetostriction present in this material. A Curie-Weiss fit of the paramagnetic regime for $T>240 \mathrm{~K}$ gives $\theta_{C W}=(115.2 \pm 0.5) \mathrm{K}$ and $\mu_{\text {eff }}=(7.85 \pm 0.01) \mu_{B} / \mathrm{Gd}$, the latter value being close to the theoretical expectation $\left(\mu_{\text {eff }}=7.94 \mu_{B} /\right.$ $\mathrm{Gd})$. This set of parameters is also consistent with the previous study of $\mathrm{Gd}_{5} \mathrm{Ge}_{4}$ which reported $\theta_{C W} \sim 94 \mathrm{~K}$ and $\mu_{\text {eff }} \sim 7.45 \mu_{B} / \mathrm{Gd}, \frac{8}{} \mathrm{~A}$ closer look at the data of Fig. 1 reveals some differences from the sample studied by Levin et $a l^{8}$, in particular a $\chi(T \rightarrow 0)$ value of the ZFC curve that is larger by $\sim 25 \%$ in our case.

The inset of Fig. 1 shows that the $\chi(T)$ curves of [PrCa40] Ga4\% exhibit low- $T$ features that are remarkably similar to those found in $\mathrm{Gd}_{5} \mathrm{Ge}_{4}$. This behavior was attributed to the appearance of a FM component in this manganite, while electron microscopy demonstrated the persistence, at low- $T$, of a short-range OO associated with the CE-type AF phase 11 The similarity between the $\chi(T)$ curves of $\mathrm{Gd}_{5} \mathrm{Ge}_{4}$ and [PrCa40] $\mathrm{Ga} 4 \%$ in the low- $T$ regime is consistent with the existence, in both systems, of related ground states based on phase separation between $\mathrm{AF}$ and FM domains.

The main panel of Fig. 2 shows a $M(H)$ curve recorded for $\mathrm{Gd}_{5} \mathrm{Ge}_{4}$ at $2 \mathrm{~K}$ after ZFC. For the field-increasing branch, there is a dramatic step in the magnetization between 2.25 and $2.50 \mathrm{~T}$. This jump is followed by a plateau, then a smooth tail, before finally reaching $M_{\text {sat }}=36.6$ $\mu_{B} /$ f.u. at $5 \mathrm{~T}$. This saturation value corresponds to $7.3 \mu_{B} / \mathrm{Gd}$, in good agreement with Ref. 8. The fielddecreasing branch is almost flat down to $\sim 1 \mathrm{~T}$ before going to zero. Increasing the field once again produces a curve superimposed on the reverse leg of the first loop, 


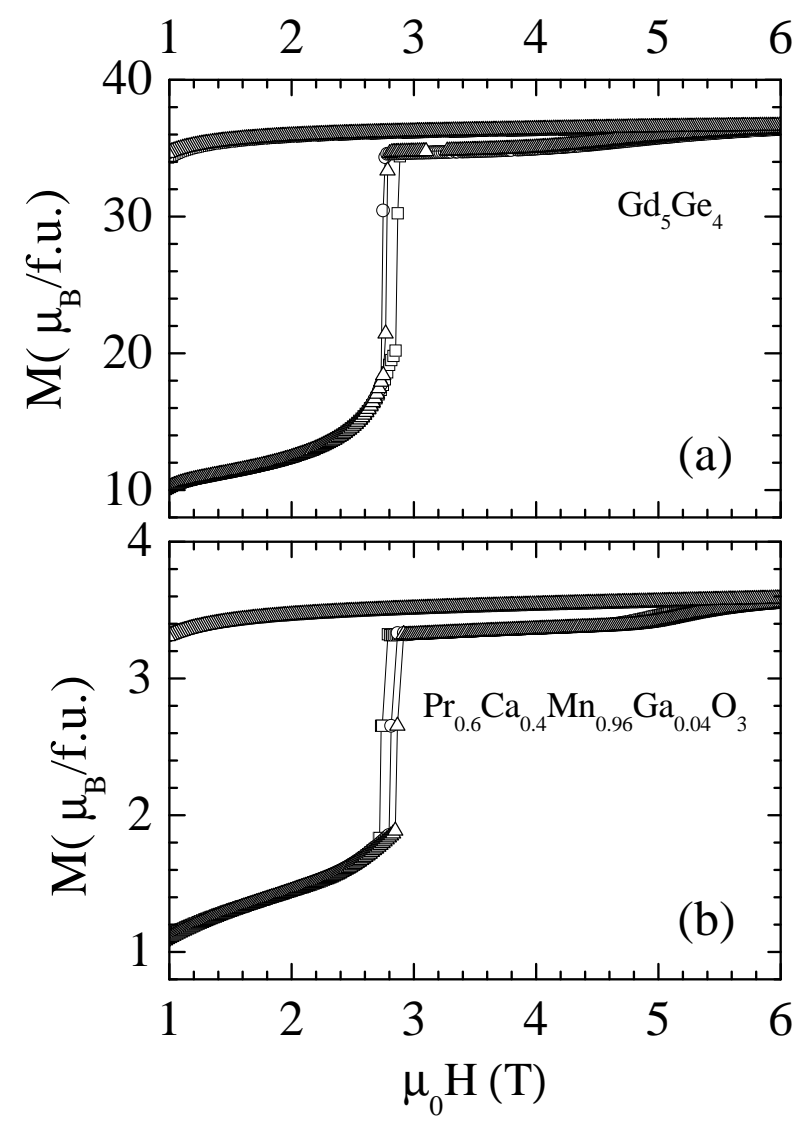

FIG. 3: Enlargements of three successive hysteresis loops recorded with a VSM, after zero-field cooling in each case: (a) $\mathrm{Gd}_{5} \mathrm{Ge}_{4}$ at $2 \mathrm{~K}$; (b) $\mathrm{Pr}_{0.6} \mathrm{Ca}_{0.4} \mathrm{Mn}_{0.96} \mathrm{Ga}_{0.04} \mathrm{O}_{3}$ at $3.25 \mathrm{~K}$. The magnetic field sweep rate is $1 \mathrm{~T} / \mathrm{min}$.

demonstrating the complete irreversibility of the transformation at this temperature. The overall behavior displayed in the main panel of Fig. 2 is in line with the features reported by Levin et al. .8 The difference, however, is that our sample exhibits a sizeable FM component, as already suggested by the ZFC value of $\chi(T \rightarrow 0)$. In Fig. 2, this is clearly revealed by the shape of the virgin magnetization curve at low fields. It is worth noting that the behavior of the sample shown in Fig. 2 is closer to that described in Ref. 8 when it is cooled in a field of 1.2 $\mathrm{T}$ to assist the onset of the FM component. We suggest that the two samples may differ on a microstructural or nanostructural level (for example, grain size or local defects), and this in turn may influence the ability of the samples to accommodate the strains associated with the martensitic (FM) phase. 14.15 .16

The inset of Fig. 2 shows a $M(H)$ curve recorded for [PrCa40] Ga4\% at $3.25 \mathrm{~K}$, after ZFC. This sample also exhibits a magnetization jump and all the features found for $\mathrm{Gd}_{5} \mathrm{Ge}_{4}$. For both compounds, the location of the magnetization steps in the $M(H)$ curves depends on the temperature. In order to obtain comparable data for each system, all the $M(H)$ loops recorded hereafter were recorded at $2 \mathrm{~K}$ for $\mathrm{Gd}_{5} \mathrm{Ge}_{4}$ and at $3.25 \mathrm{~K}$ for [PrCa40]Ga4\%.

In manganites, one of the features supporting a martensitic scenario rather than standard metamagnetism was the influence of the field spacing used to record the $M(H)$ curves by SQUID magnetometry ${ }^{13}$ It was found that smaller field increments can delay the magnetic instability, pushing the steps to higher field values. With SQUID measurements such a field-spacing effect can be related to the average magnetic field sweep rate. In the present study, we have used a VSM which is more suited to properly address this issue, since the data can be recorded while ramping the field. First of all, one must check that the compounds are not too sensitive to training effects (i.e., the shift of the step fields between successive ZFC hysteresis loops).

Figure 3 shows enlargements of three successive ZFC $M(H)$ loops recorded on $\mathrm{Gd}_{5} \mathrm{Ge}_{4}$ and $[\mathrm{PrCa} 40] \mathrm{Ga} 4 \%$. One can observe small variations from run to run in the value of the step field, which is found to be $2.78 \pm 0.08 \mathrm{~T}$ in both cases. Additional ZFC loops recorded in the case of $\mathrm{Gd}_{5} \mathrm{Ge}_{4}$ showed that the step field always lies within this range. The difference between these data and the measurements made using a SQUID magnetometer (shown in Fig. 2) will be discussed below. One should note that the scatter displayed in Fig. 3 -even though it is smallindicates that the step field of both compounds does not correspond to a well defined critical field, whereas such an history dependence is consistent with a martensitic picture. In other respects, these training effects for $\mathrm{Gd}_{5} \mathrm{Ge}_{4}$ and $[\mathrm{PrCa} 40] \mathrm{Ga} 4 \%$ remain small enough to allow a reliable investigation of the influence of the magnetic field sweep rate.

Figure 4 shows enlargements of $M(H)$ loops recorded on $\mathrm{Gd}_{5} \mathrm{Ge}_{4}$ and $[\mathrm{PrCa} 40] \mathrm{Ga} 4 \%$ with three magnetic field sweep rates $d H / d t=\dot{H}$, ranging over two orders of magnitude. In both systems, the magnetization step is found to be profoundly affected by the value of $\dot{H}$. Once again, this confirms that these step fields cannot be regarded as true critical fields for metamagnetic transitions. It appears that the influence of $\dot{H}$ is similar in both systems; as $\dot{H}$ is reduced, the smooth upturn of $M(H)$ starts at lower fields, whereas the step in the magnetization is pushed to a higher field. For $\mathrm{Gd}_{5} \mathrm{Ge}_{4}$, this effect is so pronounced that there is no longer a step for $\dot{H}=0.01 \mathrm{~T} / \mathrm{min}$. A similar disappearance of the step as $\dot{H}$ is decreased was also observed for [PrCa40] Ga4\% at $3.5 \mathrm{~K}$ for $0.1 \mathrm{~T} / \mathrm{min}$ (not shown). Such a huge impact of the magnetic field sweep rate is a feature that can be accounted for in a martensitic interpretation of the magnetization steps. Indeed, for isothermal martensitic transformations, it is known that the rate of variation of the driving force (here the magnetic field) can affect the development of the transformation. For instance, Pérez-

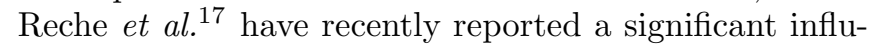
ence of the cooling rate $d T / d t$ on the temperatures of the peaks displayed on the acoustic emission spectra of 


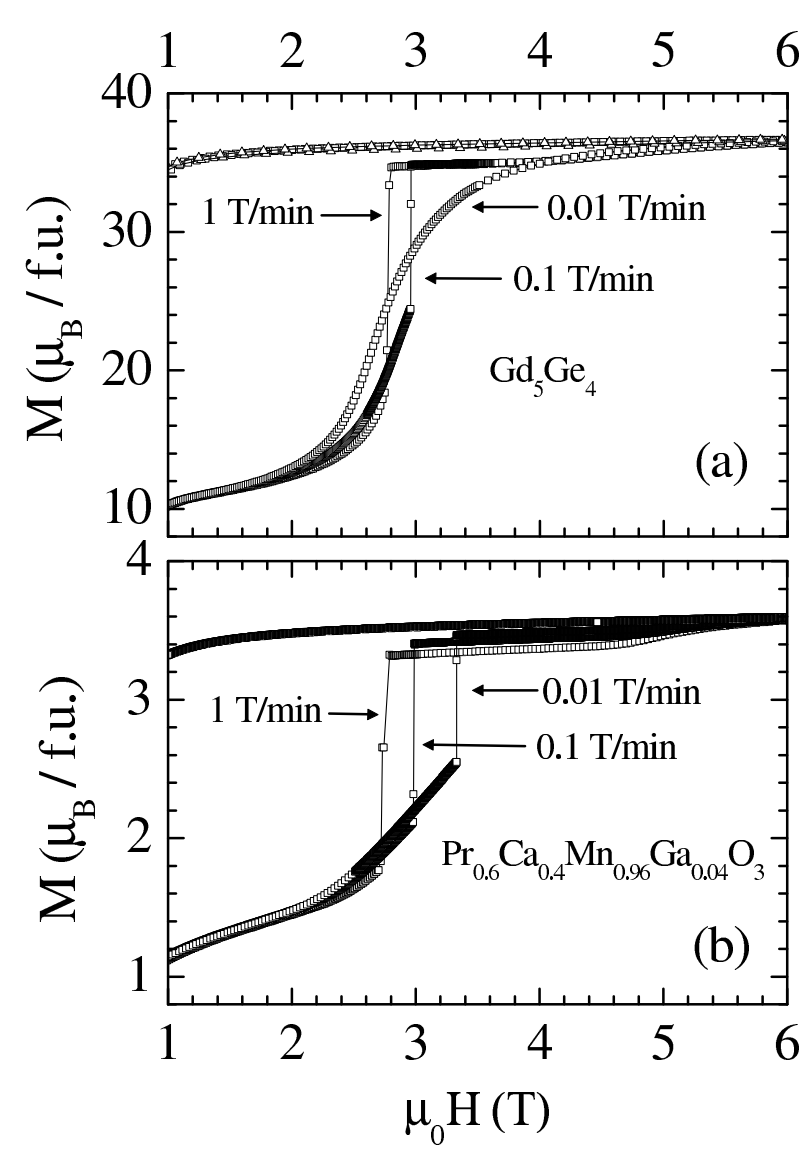

FIG. 4: Enlargements of hysteresis loops recorded with a VSM using different magnetic field sweep rates, after zero-field cooling in each case: (a) $\mathrm{Gd}_{5} \mathrm{Ge}_{4}$ at $2 \mathrm{~K}$; (b) $\mathrm{Pr}_{0.6} \mathrm{Ca}_{0.4} \mathrm{Mn}_{0.96} \mathrm{Ga}_{0.04} \mathrm{O}_{3}$ at $3.25 \mathrm{~K}$.

$\mathrm{Cu}_{68.4} \mathrm{Al}_{27.8} \mathrm{Ni}_{3.8}$. In our case, the effect is found to be more systematic and pronounced. We suggest that using a smaller $\dot{H}$ can facilitate the progressive accommodation of the martensitic strains, resulting in an upward shift of the step field and even its disappearance.
Let us now return to the comparison between the SQUID magnetometer and VSM data. According to the systematic influence of $\dot{H}$ shown by the VSM data, the locations of the step field in the SQUID data point to an effective sweep rate close to or even slightly larger than 1 $\mathrm{T} / \mathrm{min}$. For the SQUID measurements of Fig. 2, the average sweep rate (including the pause and measurements at each field) is $\sim 0.05 \mathrm{~T} / \mathrm{min}$, while the transitory sweep rate when charging the magnet is $\sim 2 \mathrm{~T} / \mathrm{min}$. Our results suggest that (i) the average sweep rate is not a relevant parameter to characterize the dynamics when the magnetic field has to be stabilized prior to each measurement; (ii) using a fast sweep rate for the field installations can play an important role in determining the response of these martensitic systems.

The present paper demonstrates that the magnetization steps recently reported for $\mathrm{Gd}_{5} \mathrm{Ge}_{4}$ have features very similar to those found in manganites like [PrCa40]Ga4\%, including a huge influence of the magnetic field sweep rate on the field-induced transformations. Such a feature is inconsistent with a standard metamagnetic transition whereas it can be qualitatively accounted for within a martensitic scenario. Although they belong to completely different classes of materials, both $\mathrm{Gd}_{5} \mathrm{Ge}_{4}$ and [PrCa40]Ga4\% turn out to be phaseseparated systems, in which FM and AF domains having very different unit cells can co-exist. Therefore, for both systems, the field-induced AF-to-FM transition at low- $T$ must be regarded as a martensitic transformation. Such transformations are well known to be discontinuous, and they can show burstlike effects. Accordingly, we propose that the similarity of the low- $T$ properties found in $\mathrm{Gd}_{5} \mathrm{Ge}_{4}$ and [PrCa40] $\mathrm{Ga} 4 \%$ is not coincidental, and that the magnetization steps are manifestations of the martensitic nature of the transformation in both systems. In this scenario, the magnetization jump corresponds to a burstlike growth of the FM component within an essentially AF matrix.

We acknowledge the financial support of the EPSRC (UK) for this project.
1 V. K. Pecharsky and K. A. Gschneidner, Phys. Rev. Lett. 78, 4494 (1997).

${ }^{2}$ L. Morellon et al., Phys. Rev. B 58, 14721 (1998).

3 L. Morellon et al., Phys. Rev. B 62, 1022 (2000).

4 J. Stankiewicz et al., Phys. Rev. B 61, 12651 (2000).

${ }^{5}$ E. M. Levin et al., Phys. Rev. B 63, 064426 (2001).

${ }^{6}$ E. M. Levin et al., Phys. Rev. B 64, 235103 (2001).

7 A. O. Pecharsky et al., J. Alloys Compd. 338, 126 (2002).

8 E. M. Levin et al., Phys. Rev. B 65, 214427 (2002).

9 J. B. Sousa et al., Phys. Rev. B 67, 134416 (2003).

10 S. Hébert et al., J. Solid State Chem. 165, 6 (2002); S. Hébert et al., Solid State Commun. 122, 335 (2002).

11 A. Maignan et al., J. Phys. Cond. Matt. 14, 11809 (2002).
12 R. Mahendiran et al., Phys. Rev. Lett. 89, 286602 (2002).

13 V. Hardy et al., J. Magn. Magn. Mater. 264, 183 (2003).

14 Z. Nishiyama, in Martensitic Transformation, edited by M. Fine et al., (Academic, New York, 1978).

15 International Conference on Martensitic Transformations (ICOMAT 95), Lausanne, 1995, edited by R. Gotthardt and J. Van Humbeeck, (Editions de Physique, Les Ulis, 1995).

16 V. Podzorov et al., Phys. Rev. B 64, 140406 (2001).

17 F. J. Perez-Reche et al., Phys. Rev. Lett. 87, 195701 (2001). 